\title{
Earth Escape from a Sun-Earth Halo Orbit using Unstable Manifold and Lunar Swingbys*
}

\author{
Hongru CHEN, ${ }^{1 \dagger} \dagger$ Yasuhiro KAWAKATSU, ${ }^{2)}$ and Toshiya HANADA ${ }^{1)}$ \\ ${ }^{1)}$ Department of Aeronautics and Astronautics, Kyushu University, Fukuoka, Fukuoka 819-0395, Japan \\ ${ }^{2)}$ Institute of Space and Astronautical Science, Japan Aerospace Exploration Agency (ISAS/JAXA), \\ Sagamihara, Kanagawa 252-5210, Japan
}

\begin{abstract}
This paper investigates the Earth escape for spacecraft in a Sun-Earth halo orbit. The escape trajectory consists of first ejecting to the unstable manifold associated with the halo orbit, then coasting along the manifold until encountering the Moon, and finally performing lunar-gravity-assisted escape. The first intersection of the manifold tube and Moon's orbit results in four intersection points. These four manifold-guided encounters have different relative velocities $\left(v_{\infty}\right)$ to the Moon; therefore, the corresponding lunar swingbys can result in different levels of characteristic energy $\left(C_{3}\right)$ with respect to the Earth. To further exploit these manifold-guided lunar encounters, subsequent swingbys utilizing solar perturbation are considered. A graphical method is introduced to reveal the theoretical upper limits of the $C_{3}$ achieved by double and multiple swingbys. The numerically solved Sun-perturbed Moon-to-Moon transfers indicate that a second lunar swingby can efficiently increase $C_{3}$. Compared to the direct low-energy escape along the manifold, applying a portion of the lunar swingbys before escape is shown to be more advantageous for deep-space mission design.
\end{abstract}

Key Words: Circular Restricted Three-body Problem, Invariant Manifolds, Luni-solar Gravity Assists, Earth Escape, Graphical Method

\section{Introduction}

The halo orbit about an equilibrium point of a three-body system (i.e., the libration point or Lagrangian point) has features such as relatively constant distances and orientation with respect to the primary and secondary bodies, which are advantageous for scientific observation and spacecraft operation. There have been several successful missions around the Sun-Earth libration points $L_{1}$ and $L_{2}$, and many are being planned. Given a small $\Delta V$, the spacecraft in a halo orbit will depart away along the invariant unstable manifold associated with the orbit. Inversely, a spacecraft along an invariant stable manifold can asymptotically converge into the corresponding halo orbit. Utilizing the manifold dynamics, interesting missions can be derived from the libration point region. In particular, low-energy escape is of great interest, and has been intensively studied. ${ }^{1-3)}$ The low-energy escape requires lower cost than the escape in the two-body model. The fuel savings during escape can contribute to an increase in payload mass. However, the energy of the spacecraft after low-energy escape only permits accessing the asteroids within a narrow zone around the Earth's orbit. Nakamiya et al. ${ }^{2}$ and Mingotti et al. ${ }^{3)}$ have shown that, for the transfer from the Earth to Mars, a $\Delta V$ of around $2 \mathrm{~km} / \mathrm{s}$ is necessary after the low-energy escape a Sun-Earth halo orbit. To gain more possibility and flexibility in interplanetary missions, escape with high energy is desirable.

Lunar swingbys have proven effective in increasing es-

(C) 2016 The Japan Society for Aeronautical and Space Sciences

*Received 18 February 2015; final revision received 1 February 2016; accepted for publication 7 April 2016.

†Corresponding author, hongru.chen@hotmail.com cape energy in some mission designs and analyses. ${ }^{4-8)}$ In particular, if a longer flight time ( $>40$ days) is permitted, solar perturbation can be utilized to vary the $v_{\infty}$ with respect to the Moon, greatly enhancing the flexibility of mission design. This strategy can be applied to improving the lunar encounter condition for gravity-assisted escape, or reducing the required insertion $\Delta v$ for a lunar mission. The mission design methods of HITEN, PLANET-B, LUNAR-A and ARTEMIS have demonstrated this technique. ${ }^{5,9,10)}$

This study analyzes the Earth escape for the spacecraft initially in a Sun-Earth $L_{1} / L_{2}$ halo orbit. It is also motivated by JAXA's Demonstration and Experiment of Space Technology for INterplanetary voYage (DESTINY) mission, which will be launched in 2019 and go to a Sun-Earth $L_{2}$ halo orbit using solar electric propulsion (SEP). ${ }^{11)}$ The possibility of extending the mission to visit a heliocentric body is currently under discussion. In order to gain sufficiently high escape energy for flexible mission design, as well as a broad reachable domain, this paper applies the associated unstable manifold for leaving the halo orbit, and then lunar swingbys for gaining energy before escape.

The paper is organized into three parts. Section 2 presents the manifold-guided lunar encounters. The Earth escape trajectories derived from the corresponding lunar swingbys are compared with direct escape along the anti-earth-ward manifold in terms of escape energy $C_{3}$ with respect to the Earth. In order to further increase $C_{3}$, Section 3 discusses the use of multiple lunar swingbys aided by solar perturbation to further increase $C_{3}$. First, a graphical method is introduced to analyze luni-solar gravity-assisted Earth escape, revealing theoretical upper limits of the $C_{3}$ achieved by double, triple and larger numbers of lunar swingbys. As considerable $C_{3}$ is 
found possible for a second swingby, Sun-perturbed Moonto-Moon transfers are numerically solved for the four types of manifold-guided encounters. Results indicate the efficiency of increasing $C_{3}$ by a second swingby, practical achievable $C_{3}$, required flight time and escape directions in this scenario. Conclusions and discussions are given in the last section.

\section{Manifold-guided Lunar Encounters for Gravity- assisted Escape}

\subsection{Halo orbits and invariant manifolds}

Trajectories are considered in the circular restricted threebody problem (CR3BP) of the Sun-Earth system. The equations of motion in the Sun-Earth synodic frame are given in Appendix. The linearized equations at the collinear equilibrium points, $L_{1}, L_{2}$ and $L_{3}$, reveal that there exist unstable periodic orbits about these points. Halo orbits are the threedimensional periodic orbits around the collinear points. Halo orbits are generally computed using numerical differential correction. ${ }^{12)}$ The monodromy matrix is the state transition matrix (STM) after one period of a periodic orbit. By examining the eigenvalues and eigenvectors of the monodromy matrix, one can know the characteristics of the orbit. For the monodromy matrix of a halo orbit, there is a pair of eigenvalues with $\lambda_{1} \cdot \lambda_{2}=1$. $^{13)}$ The dominant eigenvalue $\lambda_{1}$ is around 1500 for the Sun-Earth $L_{1} / L_{2}$ halo orbits. A small displacement along the eigenvector of the dominant eigenvalue (i.e., divergent eigenvector or unstable subspace for short) will propagate into exponentially increasing divergence from the halo orbit, since it grows $\lambda_{1}$ times in a period. Such a departure trajectory is along the invariant unstable manifold associated with the halo orbit. The manifold trajectory derived can go Earth-ward or anti-Earth-ward. Previous studies on low-energy escape utilize the anti-earthward manifolds. ${ }^{2,3)}$ In order to apply lunar gravity assists, this paper focuses on the Earth-ward manifolds. The propagation of the perturbed states yields an unstable manifold with a tube structure. Figure 1 shows the earth-ward unstable manifold trajectories originating in the Sun-Earth $L_{2}$ halo orbit with a $z$-amplitude $\left(A_{z}\right)$ of $4 \times 10^{5} \mathrm{~km}$.

\subsection{Manifold-guided lunar encounters}

This section presents the attempt to find the manifold trajectories that encounter the Moon's orbit. In this paper, the spacecraft is assumed to initially be in a Sun-Earth $L_{2}$ halo orbit. The inclination and eccentricity of the Moon's orbit are small and hence assumed to be zero, as general intersections of unstable manifolds with the Moon's orbit are considered regardless of the ephemeris. In addition, the Moon's gravity is considered only at a lunar encounter, where it impulsively deflects the $\boldsymbol{v}_{\infty}$ with respect to the Moon. Although it is a simplified model, reliable insights can still be acquired for mission design.

In Fig. 1, grey circles mark the intersections of the manifold trajectories and the ecliptic plane, outlining the intersection lines of the manifold tube and elliptic plane. It can be further observed that there are four intersections of the manifold tube and Moon's orbit. Moreover, for the wide $A_{z}$ range
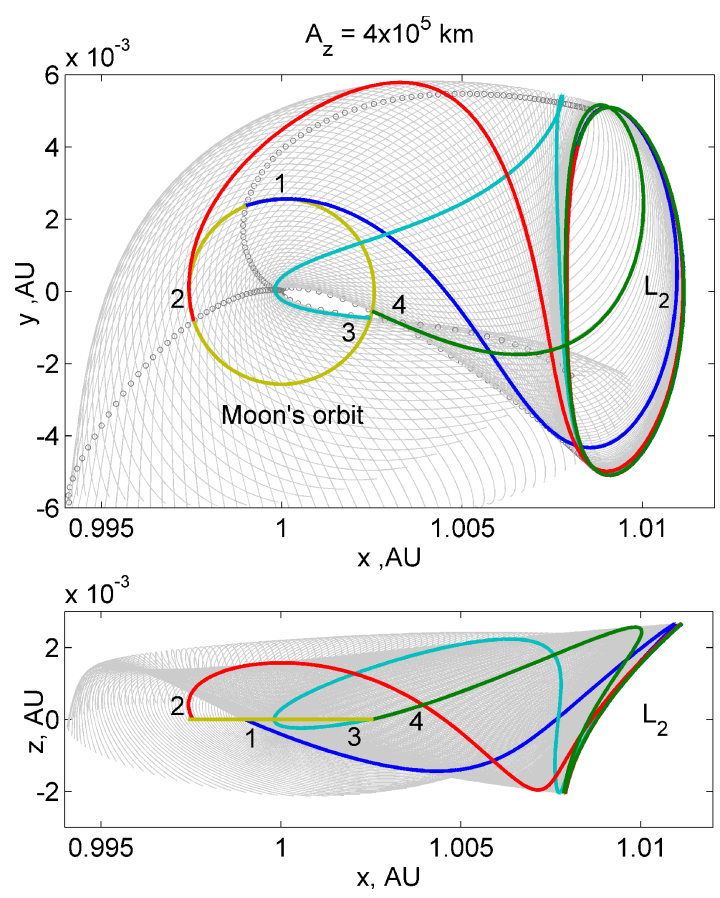

Fig. 1. The crossing of the manifold tube and the Moon's orbit exhibits four types of intersection (Sun-Earth synodic frame).

investigated (from $1 \times 10^{5} \mathrm{~km}$ to $5 \times 10^{5} \mathrm{~km}$ ), four types of intersections can be found. Note that the four intersections are found at the first crossing through the Moon's orbit. There are more intersections that take place at the second and subsequent crossings through the Moon's orbit, which would take longer flight time and are not discussed in this paper. The four manifold trajectories intersecting the Moon's orbit are obtained via interpolation. They are colored in the figures. The four types of intersections are numbered in order of the lunar phases.

Supposing the Moon will be at the intersection points when the manifold trajectories arrive at the Moon's orbit, the relative velocity $\left(v_{\infty}\right)$ to the Moon determines the lunar gravity assist capacity. Because the Jacobi integral (see Appendix) does not change too much due to small departure $\Delta V$, distances from the Moon's orbit to the Earth are identical and the Sun is relatively far, the manifold trajectories have comparable velocities with respect to the Earth at the lunar encounters. However, due to the different approaching directions with respect to the Moon's orbit, the $v_{\infty}$ differ greatly. The $v_{\infty}$ of the four types of lunar encounter are given in Fig. 2 as a function of $A_{z}$. The manifold trajectories of Type 1 and Type 2 are shown mostly tangential to the Moon's orbit while the manifold trajectories of Type 3 and Type 4 are mostly perpendicular. As a result, the encounters of Type 3 and Type 4 result in larger $v_{\infty}$ (around $1.35 \mathrm{~km} / \mathrm{s}$ ) than the encounters of Type 1 and Type 2 . The $v_{\infty}$ of Type 3 and the $v_{\infty}$ of Type 4 are nearly equal (the two lines in the figure overlap) and do not vary too much with the size of the halo orbit. The $v_{\infty}$ of Type 1 and the $v_{\infty}$ of Type 2 are comparable. They are up to $0.6 \mathrm{~km} / \mathrm{s}$ at $A_{z}=5 \times 10^{5} \mathrm{~km}$, and decrease relatively quickly as $A_{z}$ decreases. 


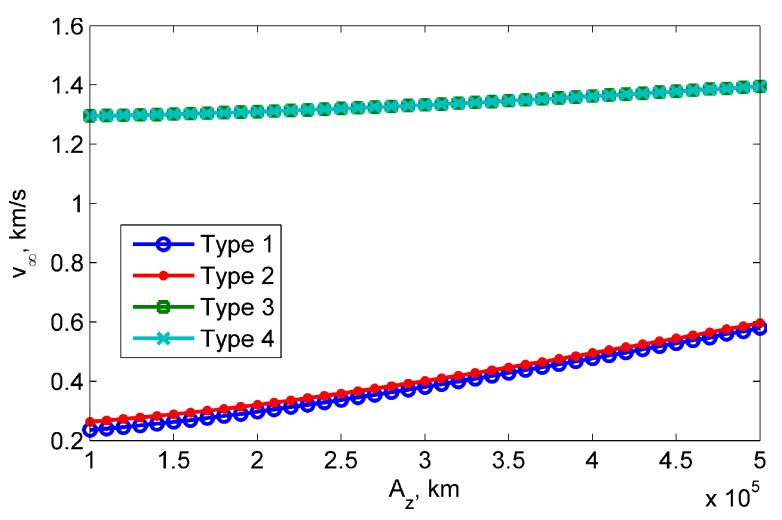

Fig. 2. $v_{\infty}$ vs. $A_{z}$ for the four types of manifold-guided lunar encounters. (The lines of Type 3 and Type 4 overlap.)

\subsection{Manifold-guided swingbys for Earth escape}

This section discusses the capacity of the four lunar encounters for Moon-gravity-assisted Earth escape. $C_{3}$ is usually used to describe the escape energy. It is twice the specific energy $\varepsilon$; that is,

$$
C_{3}=2 \varepsilon=v^{2}-2 \mu_{\mathrm{E}} / r
$$

where $\mu_{\mathrm{E}}$ is the gravitational parameter of the Earth, and $v$ and $r$ are the velocity and position with respect to the Earth. For brevity, unless noted, $C_{3}$ presented hereafter refers to $C_{3}$ with respect to the Earth, and $v_{\infty}$ refers to $v_{\infty}$ with respect to the Moon. As $C_{3}$ can be influenced by the solar perturbation, $C_{3 \mathrm{LSB}}$ is used to represent the osculating $C_{3}$ at the lunar swingby. The radius of the Moon's orbit $r_{M}$ is assumed to be constant $(=384,400 \mathrm{~km})$. In order to get the maximum post-swingby $C_{3 \mathrm{LSB}}, C_{3 \mathrm{LSB}}{ }^{+}$max , the post-swingby velocity $v^{+}$(superscripts "-," and "+ " indicate the states prior to and after a swingby, respectively) should be maximized. A lunar swingby will rotate the incoming $\boldsymbol{v}_{\infty}{ }^{-}$by angle $\delta$ to outgoing $\boldsymbol{v}_{\infty}{ }^{+}$. The bending angle $\delta$ is associated with the perilune altitude, which can be targeted by an infinitesimal maneuver at a far distance before the swingby. In order to obtain $v^{+}$max , the outgoing $\boldsymbol{v}_{\infty}{ }^{+}$should be aligned with the Moon's velocity vector as much as possible. However, $\delta$ is limited to the $\delta_{\max }$, expressed by

$$
\delta_{\max }=\pi-2 \times \arccos \left[\mu_{\mathrm{M}} /\left(\mu_{\mathrm{M}}+r_{p \min } v_{\infty}{ }^{2}\right)\right]
$$

where $\mu_{\mathrm{M}}$ is the gravitational parameter of the Moon, $r_{p \text { min }}$ is the minimum perilune (i.e., the sum of the Moon's radius $1738 \mathrm{~km}$ and the minimum permissible flyby altitude, which is $100 \mathrm{~km}$ in this study). If the pump angle $\varphi^{-}$between $\boldsymbol{v}_{\infty}{ }^{-}$ and the Moon's velocity $\boldsymbol{v}_{\mathrm{M}}$ is smaller than $\delta_{\max }, v^{+}{ }_{\max }$ is the sum of the $v_{\mathrm{M}}$ and $v_{\infty}$ magnitudes. Otherwise, to obtain $v^{+}{ }_{\max }, \boldsymbol{v}_{\infty}{ }^{-}$should be rotated by $\delta_{\max }$ to approach $\boldsymbol{v}_{\mathrm{M}}$ in the plane determined by $\boldsymbol{v}_{\infty}{ }^{-}$and $\boldsymbol{v}_{\mathrm{M}}$. The two situations are illustrated in Fig. 3. The expression of $v^{+}{ }_{\max }$ is,

$v_{\text {max }}^{+}=\left\{\begin{array}{lr}v_{M}+v_{\infty} & \left(\varphi^{-} \leq \delta_{\max }\right) \\ \sqrt{v_{M}^{2}+v_{\infty}^{2}+2 v_{M} v_{\infty} \cos \left(\varphi^{-}-\delta_{\max }\right)} & \left(\varphi^{-}>\delta_{\max }\right)\end{array}\right.$
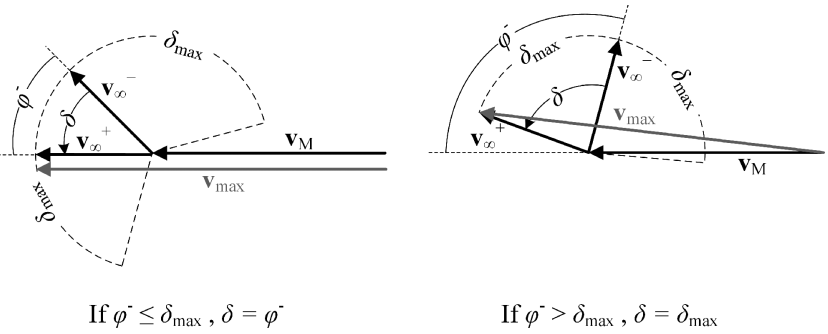

Fig. 3. Use lunar swingbys to bend $\boldsymbol{v}_{\infty}$ to achieve the maximum postswingby velocities.

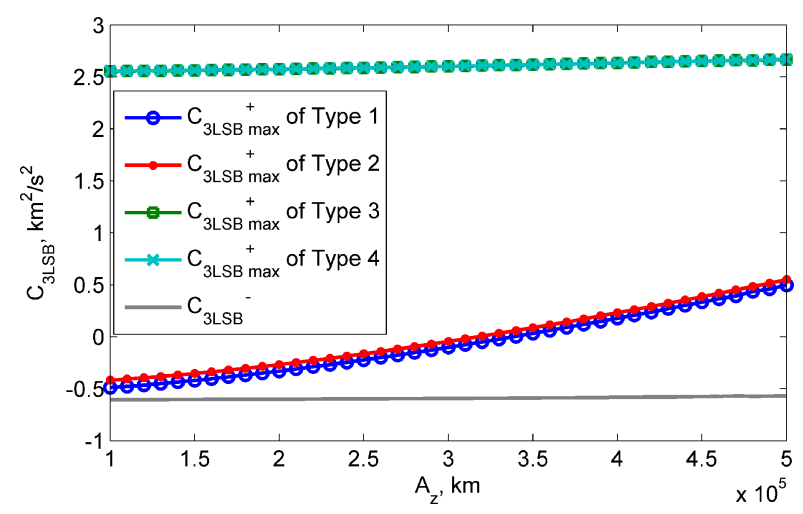

Fig. 4. $C_{3 \mathrm{LSB}}{ }^{-}$and $C_{3 \mathrm{LSB}}{ }^{+}{ }_{\text {max }}$ for the four types of lunar swingby. (The lines of Type 3 and Type 4 overlap.)

Then, one can acquire the capacity of the lunar encounter for gravity-assisted Earth escape, which is expressed by,

$$
C_{3 \mathrm{LSB}}{ }^{+}{ }_{\max }=v^{+}{ }_{\max }{ }^{2}-2 \mu_{\mathrm{E}} / r_{\mathrm{M}}
$$

The $C_{3 \mathrm{LSB}}{ }^{+}$max for each type of lunar swingby as a function of $A_{z}$ is shown in Fig. 4. As explained earlier, the velocities of the manifold trajectories with respect to the Earth at lunar encounters are comparable, and so is $C_{3 \mathrm{LSB}}{ }^{-}$(i.e., $C_{3}$ before lunar swingby). For comparison, $C_{3 \mathrm{LSB}}{ }^{-}$is also displayed in the figure. Without lunar swingbys, the manifold trajectories are considered non-escape as $C_{3 \mathrm{LSB}}{ }^{-}$is below zero. Similar to the profiles of $v_{\infty}$, the $C_{3 \mathrm{LSB}}{ }^{+}$max of Type 3 and Type 4 do not vary too much with $A_{z}$, around $2.6 \mathrm{~km}^{2} / \mathrm{s}^{2}$. The $C_{3 \mathrm{LSB}}{ }^{+}{ }_{\max }$ of Type 1 and Type 2 are much smaller, with $0.5 \mathrm{~km}^{2} / \mathrm{s}^{2}$ at $A_{z}=5 \times 10^{5} \mathrm{~km}$. Furthermore, the swingbys of Type 1 and Type 2 cannot lead to Earth escape if $A_{z}$ is smaller than $3 \times 10^{5} \mathrm{~km}$ (however, the solar perturbation might change the escape situation slightly; see next subsection).

\subsection{Comparison with direct escape along the anti- Earth-ward manifold}

As has been mentioned, by varying the bending angle $\delta$ within $\delta_{\max }$, one can get various post-swingby states as well as different $C_{3 \mathrm{LSB}}{ }^{+}$. However, the Sun also influences the trajectory, especially at the edge of the Earth's sphere of influence. A trajectory with low positive $C_{3 \mathrm{LSB}}{ }^{+}$could be decelerated and cannot escape for a long time. To examine escape directions, the post-swingby states are propagated until the Earth's gravity is negligible. For this purpose, a sphere of escape judgment (SoE) centered at the Earth is defined with a 


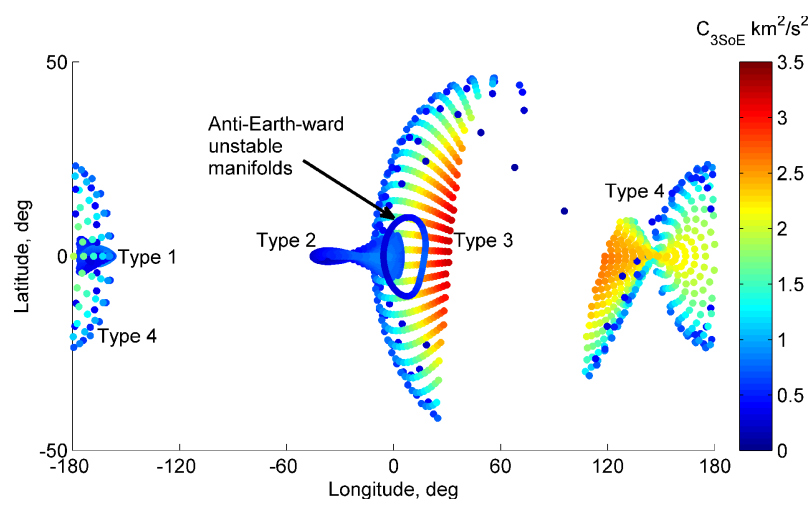

Fig. 5. The $C_{3 \mathrm{SoE}}$ magnitudes and escape directions for the manifoldguided lunar swingbys and direct escape along the anti-earth-ward unstable manifold.

radius of $0.02 \mathrm{AU}^{\ddagger}$ (i.e., approximately two times the Earth$L_{2}$ distance). At this distance, the Earth's gravity is less than one tenth of the Sun's. If a post-swingby trajectory cannot reach the SoE in a sufficiently long time (i.e., 60 days in this study), it is considered non-escape. Furthermore, the escape due to a lunar swingby can be compared with the direct escape along the anti-Earth-ward unstable manifold. In the following, the halo orbit with $A_{z}=4 \times 10^{5} \mathrm{~km}$ is taken as an example for further discussion. The $C_{3}$ magnitudes at the SoE, $C_{3 \mathrm{SoE}}$, and escape directions represented by longitudes and latitudes with respect to the Sun-Earth ecliptic coordinate are printed in Fig. 5. The enclosed solid circle in the figure indicates direct escape along the anti-Earth-ward manifold. The dots indicate post-swingby trajectories acquired by varying $\delta$. The $C_{3 \mathrm{SoE}}$ of direct manifold escape ranges from 0.3 to $0.7 \mathrm{~km}^{2} / \mathrm{s}^{2}$. The maximum $C_{3 \mathrm{SoE}}$ of the post-swingby trajectories is $3.3 \mathrm{~km}^{2} / \mathrm{s}^{2}$. The increase from the maximum $C_{3 \mathrm{LSB}}{ }^{+}{ }_{\text {max }}$ of $2.6 \mathrm{~km}^{2} / \mathrm{s}^{2}$ is due to solar perturbation. Higher $C_{3 \mathrm{SoE}}$ suggests a lager reachable domain in deep space, as well as more accessible targets. The lunar swingbys lead to a wider range of escape directions as well.

\section{Multiple Lunar Swingbys for Earth Escape}

In the preceding section, the lunar swingbys of Type 1 and Type 2 are found to be ineffective for Earth escape. In order to sufficiently utilize these manifold-guided lunar encounters, subsequent lunar swingbys are considered. Under the two-body model, multiple lunar swingbys have invariant $\boldsymbol{v}_{\infty}$ with respect to the Moon. However, solar gravitational perturbation (i.e., solar tidal force) can vary $\boldsymbol{v}_{\infty}$ if the loop between the two lunar swingbys reaches the region where solar perturbation is significant. The solar tidal force in the Earthcentered inertial frame and its effect on orbits around the Earth are schematically depicted in Fig. 6. As illustrated, after the first lunar swingby (S1), a consequent orbit with its apogee far away in 1st or 3rd quadrants will experience posigrade deceleration near the apogee, and come back to

${ }^{\ddagger}$ Astronomical Unit, $1 \mathrm{AU}=1.5 \times 10^{8} \mathrm{~km}$ (i.e., mean Sun-Earth distance).

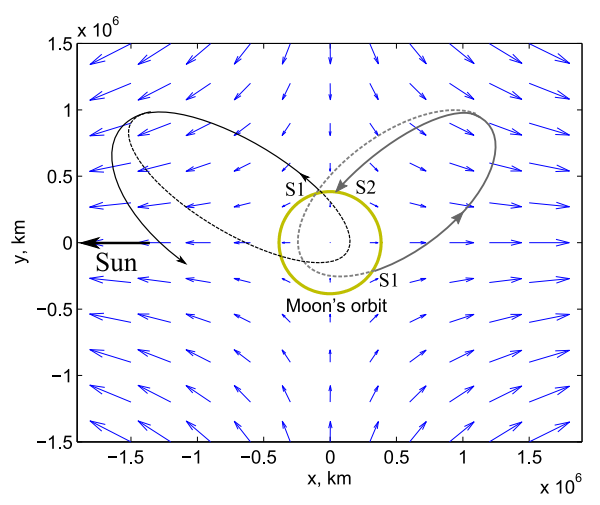

Fig. 6. The solar tidal force and its influence on Earth orbits (Earth-centered inertial frame).

a second lunar swingby (S2) with a larger encounter angle with respect to the Moon or even in a retrograde direction, resulting in an increased $v_{\infty}$. On the contrary, an orbit with its apogee in 2nd or 4th quadrants will be accelerated, resulting in a decreased $v_{\infty}$ at S2 or missing the Moon's orbit. Through experiments, it is observed that, when the osculating apogee $r_{a}$ at S1 is greater than $8 \times 10^{5} \mathrm{~km}$, solar perturbation can significantly change the shape of the postswingby orbit and the re-encounter $v_{\infty}$.

Section 3.1 presents a graphical analysis. The graph shows insights into the influence of lunar swingby and Sun-perturbed transfer on the capacity of a subsequent lunar swingby for Earth escape in terms of $C_{3 \mathrm{LSB}}{ }^{+}{ }_{\max }$. The graphical analysis can reveal (a) the upper limit of $C_{3 \mathrm{LSB}}{ }^{+}$max of the second swingby, based on the condition of the first swingby; (b) the upper limits of the $C_{3 \mathrm{LSB}}{ }^{+}{ }_{\text {max }}$ achieved by short swingbys, double swingbys and swingbys occurring in larger numbers. The upper limit is a quick assessment of the subsequent swingbys for Earth escape without numerically solving the Moon-to-Moon transfers. The planar Moon-to-Moon transfers are solved for the four manifold-guided encounters using the routine presented in Section 3.2.

\subsection{Swingby-Jacobi graph}

Given a set of $v_{\infty}$ and $\varphi$ between the $\boldsymbol{v}_{\infty}$ and $\boldsymbol{v}_{\mathrm{M}}$, one can compute the variables at lunar swingby, such as osculating velocity $\boldsymbol{v}$, radius of apogee $r_{a}$, specific energy $\varepsilon$ (i.e., half of $C_{3 \mathrm{LSB}}$ ), and encounter angle $\alpha$ between $\boldsymbol{v}$ and $\boldsymbol{v}_{\mathrm{M}}$. In addition, the three-body energy Jacobi integral $J$ in the CR3BP can be computed based on $v$ and an arbitrary position in the Moon's orbit. Although $J$ varies with the position along the Moon's orbit and velocity direction, it only varies within a negligible range. Note that these variables can refer to preor post-swingby states using superscripts “-” or "+". Recalling Eqs. (3) and (4), $C_{3 \mathrm{LSB}}{ }^{+}{ }_{\max }$ is determined by $v_{\infty}$ and angle $\varphi^{-}$, i.e. $\mathrm{S}^{-}$. Figure 7 gives contours of these variables on the $v_{\infty}-\varphi$ plane.

A zoom-in view of Fig. 7 is shown in Fig. 8 to explain the luni-solar gravitational influence on $C_{3 \mathrm{LSB}}{ }^{+}{ }_{\max }$. By a swingby, $v_{\infty}$ remains constant while $\varphi^{-}$changes to $\varphi^{+}$. To illustrate, in Fig. 8, a pre-swingby state $a_{\mathrm{S} 1}{ }^{-}$jumps vertically to a different $\varphi^{+}$levels as the result of a swingby. The con- 


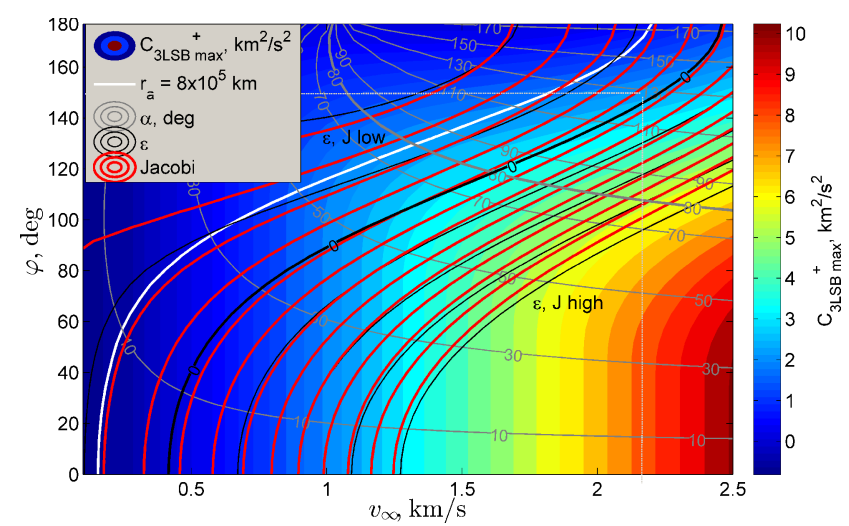

Fig. 7. Variations of encounter angle $\alpha, C_{3 \mathrm{LSB}}{ }^{+}$max , specific energy $\varepsilon$ and Jacobi integral $J$ on the $v_{\infty}-\varphi$ plane.

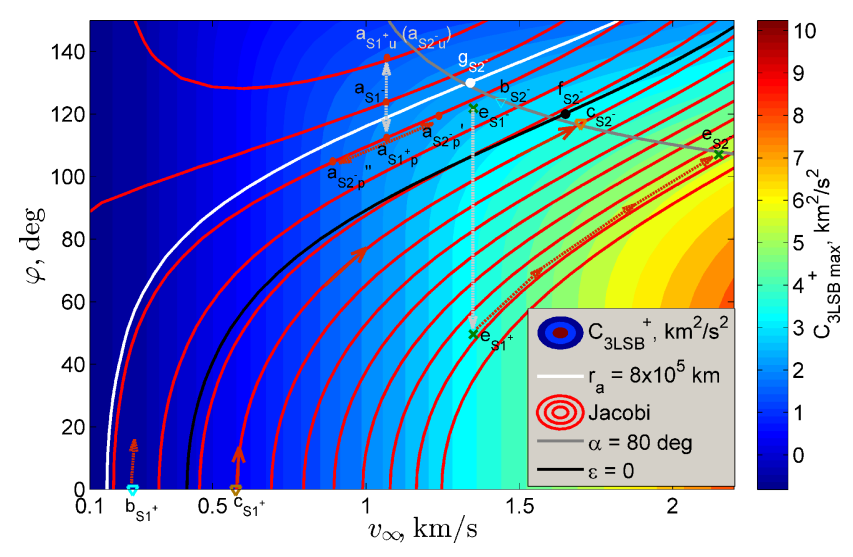

Fig. 8. State changes due to lunar swingbys and Sun-perturbed transfers.

tour of $r_{a}=8 \times 10^{5} \mathrm{~km}$ is drawn to separate the regions where solar perturbation is insignificant (upper) and significant (lower). If the post-swingby state is below this contour, such as $a_{\mathrm{S} 1}{ }^{+} \mathrm{P}$ in the figure, the subsequent $\mathrm{S} 1$-to-S2 (i.e., Moon-to-Moon) transfer is perturbed by the Sun, but $J$ is maintained. Therefore, the state will slide along the contour of the same $J$ level during the transfer, and terminate at an $\mathrm{S} 2{ }^{-}$, such as $a_{\mathrm{S} 2}{ }^{-} \mathrm{p}^{\prime}$ and $a_{\mathrm{S} 2}{ }^{-} \mathrm{p}^{\prime \prime}$. Along a $J$ contour towards the direction of $v_{\infty}$ increase, $\varepsilon$ decreases, suggesting deceleration by the Sun, and $\alpha$ increases, reflecting solar influence on orbits' shape, as is shown in Fig. 6. If the post-swingby state is above the $r_{a}=8 \times 10^{5} \mathrm{~km}$ contour, such as $a_{\mathrm{S} 1}{ }_{\mathrm{u}}$, which suggests a subsequent nearly-unperturbed transfer, $v_{\infty}$ will not change due to the transfer, thus $\mathrm{S}^{-} \approx \mathrm{S} 1^{+}$. The $\mathrm{S} 2^{-}$state reflects the $C_{3 \mathrm{LSB}}{ }^{+}$max of the second swingby. In summary, the graph can display the influence of the Moon (vertical motion) and Sun (motion along a $J$ contour) on $C_{3 \mathrm{LSB}}{ }^{+}$max. This graph is referred to as the swingby-Jacobi graph for conciseness.

For unperturbed transfers, no matter how many times lunar swingbys are performed, as $v_{\infty}$ is invariant, the variation of $C_{3 \mathrm{LSB}}{ }^{+}$max is confined by vertical motion on the graph. The upper limit of the $C_{3 \mathrm{LSB}}{ }^{+}{ }_{\max }$ for various $v_{\infty}$ is found to be $2.3 \mathrm{~km}^{2} / \mathrm{s}^{2}$ at the tangent point of the contours of $r_{a}=8 \times 10^{5} \mathrm{~km}$ and the contours of $C_{3 \mathrm{LSB}}{ }^{+}$max $\left(g_{\mathrm{S} 2^{-}}\right)$. To further increase $C_{3 \mathrm{LSB}}{ }^{+}$max , the region affected by solar per-

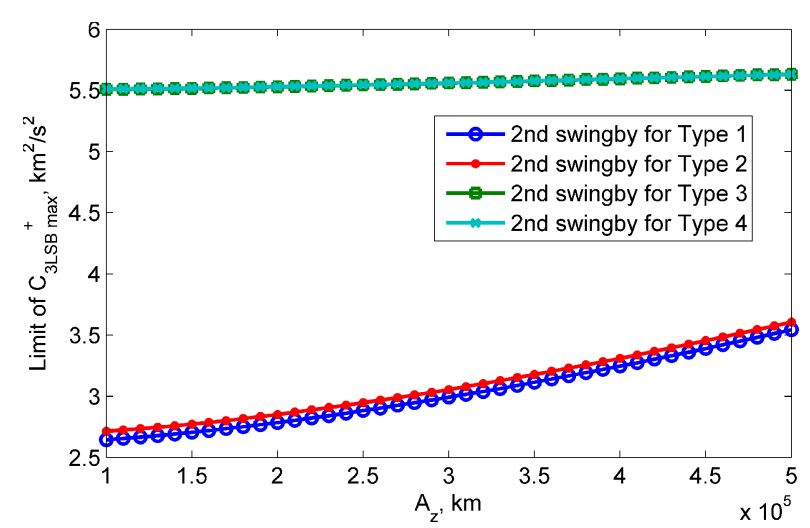

Fig. 9. Without flight time constraint, the limit of $C_{3 \mathrm{LSB}}{ }^{+}$max of the second swingby for the four types of first swingbys (i.e., manifold-guided swingbys).

turbation should be explored.

Inspecting Fig. 7, the contours of $J$ are shown tangential to the contours of $C_{3 \mathrm{LSB}}{ }^{+}$max at $\alpha \approx 80^{\circ}$. High $\alpha$ results in high $v_{\infty}$, but limited bending angle. $\alpha=80^{\circ}$ appears to be the optimal encounter condition for a post-swingby trajectory to get the largest $C_{3 \mathrm{LSB}}{ }^{+}{ }_{\max }$. If the new $\mathrm{S}^{-}$state approaches $\alpha=80^{\circ}$, such as to $a_{\mathrm{S} 2}{ }^{-}{ }^{\prime}, C_{3 \mathrm{LSB}}{ }^{+}{ }_{\max }$ will increase. Oppositely, if the state slides away from $\alpha=80^{\circ}$, such as to $a_{\mathrm{S} 2}{ }^{-} 2^{\prime \prime}, C_{3 \mathrm{LSB}}{ }^{+}{ }_{\max }$ will decrease. In addition, along the $\alpha=80^{\circ}$ level, $C_{3 \mathrm{LSB}}{ }^{+}{ }_{\text {max }}$ increases with $J$. Then, one can estimate the upper limit of $C_{3 \mathrm{LSB}}{ }^{+}{ }_{\text {max }}$ of $\mathrm{S} 2$ based on the $\mathrm{S} 1$ state. Considering the manifold-guided $\mathrm{S} 1$, for Type 1 and Type 2 , the $v_{\infty}$ ranges from 0.24 to $0.6 \mathrm{~km} / \mathrm{s}$ for the range considered for $A_{z}$ (see Fig. 2). Points $b_{\mathrm{S} 1^{+}}$and $c_{\mathrm{S} 1^{+}}$locate $\varphi^{+}=0^{\circ}$, and thus maximize $J^{+}$for the two $v_{\infty}$ boundaries. Along the $J$ contours linking with $b_{\mathrm{S} 1^{+}}$and $c_{\mathrm{S} 1^{+}}$, the upper limit of $C_{3 \mathrm{LSB}}{ }^{+}{ }_{\max }$ are found to be 2.6 and $3.7 \mathrm{~km}^{2} / \mathrm{s}^{2}$ at $\alpha \approx 80^{\circ}\left(b_{\mathrm{S} 2^{-}}\right.$and $\left.c_{\mathrm{S} 2^{-}}\right)$. For Type 3 and Type 4 , the $v_{\infty}$ is around $1.35 \mathrm{~km} / \mathrm{s}$ and $\varphi^{-}$is $120^{\circ}$. The $v_{\infty}$ magnitude constrains the bending angle. It can be computed that, $\varphi^{+}>50^{\circ}$ for Type 3 and Type 4 . Then, the upper limit of $C_{3 \mathrm{LSB}}{ }^{+}$max along the $J$ level that links with the state $e_{\mathrm{S} 1^{+}}(1.35,50)$ is $5.6 \mathrm{~km}^{2} / \mathrm{s}^{2}\left(e_{\mathrm{S}_{2}}\right)$. The upper limit of $C_{3 \mathrm{LSB}}{ }^{+}$max of the $\mathrm{S} 2$ for the four types of $\mathrm{S} 1$ is shown as a function of $A_{z}$ in Fig. 9. The limitation of the swingby-Jacobi graph should be noted. The lunar phase of $\mathrm{S} 1$, which defines the initial condition along with $v_{\infty}$ and $\varphi^{-}$, and flight time are not specified in the graph. Therefore, not every $\mathrm{S} 2^{-}$state along the $J$ level can be explored from the $\mathrm{S}^{+}$state within a limited flight time. Nevertheless, given no flight time constraint, it is reasonable to assume that every state on the $J$ level can be reached. Therefore, the upper limits obtained from the graph are the theoretical maxima that should not be exceeded in the real world.

Since flight time is critical in space missions, it is limited to 200 days for further discussions. If the spacecraft departs to a hyperbolic orbit $(\varepsilon>0)$ at $\mathrm{S} 1$, it will probably escape. In that case, orbits such as resonance orbits with the Earth and SunEarth Lyapunov orbits that are large enough to intersect with the Moon's orbit are possible Moon-to-Moon transfer solutions. But these orbits require significant flight time generally 


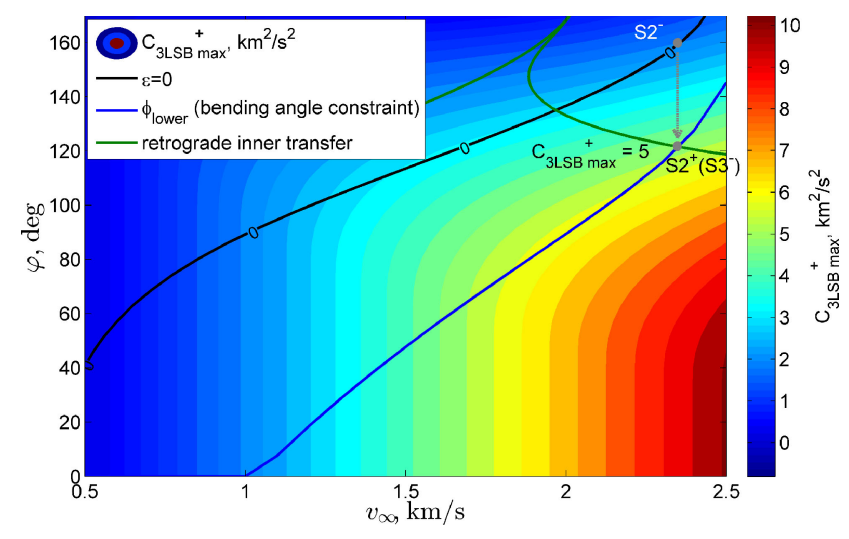

Fig. 10. Obtaining $C_{3 \mathrm{LSB}}{ }^{+}{ }_{\max }$ of a triple lunar swingby.

longer than 200 days. With the fight time constraint, S1-to-S2 transfers are considered as perturbed elliptic transfers. Then, the state change is bound by the contour with $\varepsilon=0$. Hence, the limit of $C_{3 \mathrm{LSB}}{ }^{+}{ }_{\text {max }}$ of the second lunar swingby would be around $3.3 \mathrm{~km}^{2} / \mathrm{s}^{2}$, which is acquired at the tangent point of the contours of $\varepsilon=0$ and $C_{3 \mathrm{LSB}}{ }^{+}{ }_{\max }\left(f_{\mathrm{S}_{2}-}\right)$. In addition, if the second swingby delivers the spacecraft to a retrograde orbit, the spacecraft can encounter the Moon once more on the outbound leg, as is demonstrated in Kawaguchi et al. ${ }^{5)}$ and McElrath et al. ${ }^{14)}$ The solutions of $\varphi^{+}$and $v_{\infty}$ to retrograde transfers are obtained by solving the Lambert problem for varied positions along the Moon's orbit and the corresponding flight time. The solution sets are represented by the green line in Fig. 10. The last second swingby can shift $\mathrm{S} 2^{-}$to the $\mathrm{S}^{+}$along the green line for a retrograde transfer to the Moon (S3). As the consequent S2-to-S3 transfer is within the Moon's orbit, solar perturbation cannot significantly change the encounter state. Hence, $\mathrm{S}^{-}=\mathrm{S} 2^{+}$. As is shown, states along the green line upon $\varepsilon=0$ suggest hyperbolic transfers, which open up a pathway to increase $C_{3 \mathrm{LSB}}{ }^{+}{ }_{\max }$. However, the bending angle limit sets a constraint. The lower boundaries of $\varphi^{+}$are computed for various $\mathrm{S}^{-}$states along the $\varepsilon=0$ contour, which are represented by the blue line in Fig. 10. The upper limit of $C_{3 \mathrm{LSB}}{ }^{+}$max for the third swingby is found to be $5 \mathrm{~km}^{2} / \mathrm{s}^{2}$ at the intersection of the lines of the retrograde transfer solution and lower boundary of $\varphi^{+}$. Note that this upper limit of $5 \mathrm{~km}^{2} / \mathrm{s}^{2}$ also applies to other cases with larger numbers of swingbys.

\subsection{Solve for Sun-perturbed Moon-to-Moon transfers}

According to Fig. 9, a second lunar swingby might increase $C_{3 \mathrm{LSB}}$ to a considerable level of $3.3 \mathrm{~km}^{2} / \mathrm{s}^{2}$ in 200 days. The second lunar swingbys for the manifold-guided $\mathrm{S} 1$ are to be solved. This subsection presents the routine of solving the planar S1-to-S2 transfers for a given S1 condition.

The initial condition of an S1-to-S2 transfer is defined by the direction of $\boldsymbol{v}_{\infty}{ }^{+}$. In the planer case, longitude angle $\beta$ with respect to the Earth-Moon axis is used to specify the direction of $\boldsymbol{v}_{\infty}{ }^{+}$. After a time of flight (ToF), the distance to the Moon's orbit $\Delta d$ and phase difference $\Delta \theta$ from the lunar phase should be zero for a re-encounter event. $\beta$ and $T o F$ determine whether the spacecraft re-encounters the Moon. Namely, the solution set is,

$$
\{(\beta, T o F) \mid \Delta \theta=0 \wedge \Delta d=0\}
$$

In searching the solutions, $\beta$ is changed from $0^{\circ}$ to $360^{\circ}$ in an initial increment of $0.05^{\circ}$. The initial conditions that cannot have osculating $r_{a}$ greater than $8 \times 10^{5} \mathrm{~km}$ are excluded from the search. Otherwise, there will be solutions of resonances with the Moon, which are not the major concern here and can be solved in a simpler way. The initial state of given $\beta$ is propagated until the trajectory reaches the Moon's orbit from outside. Thus, at termination, the trajectory has passed through the apogee where solar perturbation exerts the greatest influence on the trajectory. Moreover, this step imposes $\Delta d=0$ and can return the corresponding $T o F$ and $\Delta \theta$. Then, the problem is simplified to locating $\Delta \theta=0$. If the sign of $\Delta \theta$ changes at two consecutive samples, the interval between the two samples should include a solution. Close guesses of solutions are obtained by interpolating $\beta$ and $T o F$ at $\Delta \theta=0$ in the solution-including intervals. The last step is to get the accurate $\beta$ and ToF by performing differential corrections to target the Moon's position. Note that the propagation time is limited to 200 days. Solutions in the $\beta$ intervals that cannot lead to a comeback to the Moon's orbit in 200 days are excluded from discussion. In addition, the $\beta$ intervals that are not inside the bendable angle region are also excluded.

On the other hand, $\Delta \theta$ becomes very sensitive to $\beta$ as the period of the post-swingby orbit increases. A tiny change of $\beta$ can result in a great shift in $\Delta \theta$. Consequently, the true $\Delta \theta$ variation cannot be captured by the fixed step-size sampling. To cope with this, the step size of $\beta$ is adjusted to maintain the changes of $\Delta \theta$ to be smaller than $30^{\circ}$. The resolution of $\beta$ is down to $0.007^{\circ}$ for the longest ToF cases, and an increment as great as $1.5^{\circ}$ is found sufficient for the shortest ToF cases. With the step-size control, the algorithm becomes efficient and unlikely to miss solutions.

In addition, there is another case of lunar encounter. The one passing the Moon's orbit on the inbound leg (inbound case) is already considered. The other one passes the Moon's orbit from inside (outbound case). The outbound case can be easily derived from the states at the inbound crossing as the trajectory has come to the near-Earth realm where the Keplerian elements can be applied to obtain $\Delta \theta$ and $T o F$ at the outbound crossing. Moreover, there are also cases that the trajectory passes the Moon's orbit several times before a re-encounter. To find multiple-loop solutions, the program is modified to identify all inbound crossings and return the corresponding $\Delta \theta$ and $T o F$ variations for each inbound and outbound crossing. Lantoine and McElrath ${ }^{15}$ ) have solved for Moon-to-Moon transfers for various $v_{\infty}$, flight time and orbit types, using grid search and continuation method. However, the multiple-loop Moon-to-Moon transfers are not presented in that work.

The routine described in this paper is executed using Matlab R2012a on a computer equipped with the following: CPU, Intel i7-3770 @ $3.4 \mathrm{GHz}$ and 8 GB of RAM. For a given $\mathrm{S} 1$, the program generally finds all Sun-perturbed Moon-to-Moon solutions in $2 \mathrm{~min}$. 

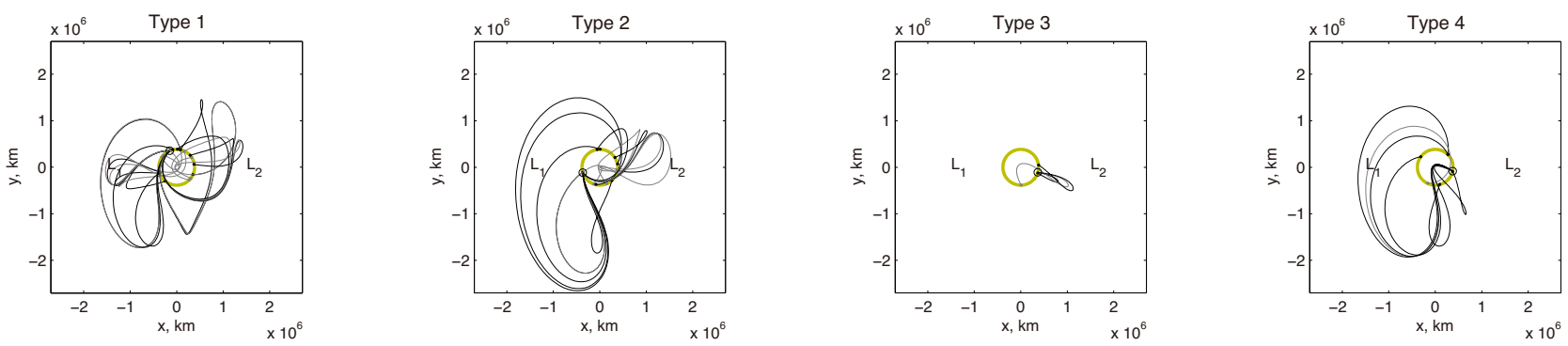

Fig. 11. Sun-perturbed Moon-to-Moon transfers found for each type of manifold-guided encounter.

Inbound and outbound solutions are displayed in dark and light colors, respectively. S1 are marked by circles and S2 by dots.

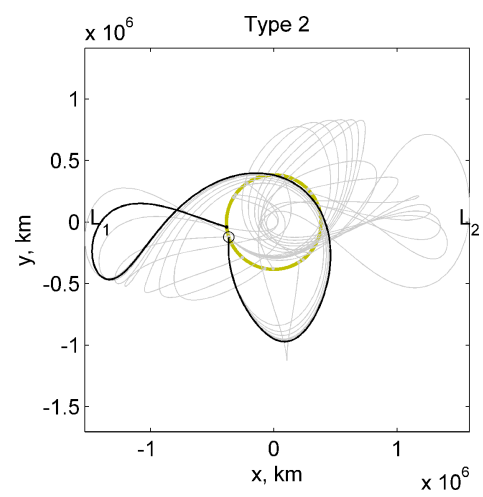

Fig. 12. Moon-to-Moon transfers with two Sun-perturbed loops for the S1 of Type 2 .

S1 are marked by circles and S2 by dots.

\subsection{Results and discussions}

The S1-to-S2 transfers solved for each type of manifoldguided encounter for $A_{z}=4 \times 10^{5} \mathrm{~km}$ are presented here. Because the problem of interest is high- $C_{3}$ Earth escape, the solutions with $v_{\infty}$ lower than $0.5 \mathrm{~km} / \mathrm{s}$ at $\mathrm{S} 2$ are not presented. Figure 11 shows the transfers with one Sun-perturbed loop. Because the transfers shown result in increased $v_{\infty}$ at $\mathrm{S} 2$ for Type 1 and Type 2, the trajectories are decelerated by the Sun. The apogees of the transfers for Type 1 and Type 2 are generally in 1st or 3rd quadrants accordingly. Figure 12 shows the two-loop transfers for Type 2 as an example, with one solution highlighted. It can be seen that, after one loop, the apogees shift to 1st or 3rd quadrant. Within 200 days, the solutions are found to be up to three loops for the S1 of Type 3 and Type 4, and two loops for the S1 of Type 1 and Type 2 .

The $C_{3 \mathrm{LSB}}{ }^{+}{ }_{\max }$ of the $\mathrm{S} 2$ vs $T o F$ is plotted in Fig. 13. The maximum $C_{3 \mathrm{LSB}}{ }^{+}{ }_{\max }$ of $\mathrm{S} 2$ found in 200 days is $2.7 \mathrm{~km}^{2} / \mathrm{s}^{2}$ for all types. The $\mathrm{S} 2$ for the $\mathrm{S} 1$ of Type 1 and Type 2 achieve this practical maximum level in 95 and 75 days, respectively. However, the second swingby for Type 3 and Type 4 cannot further increase $C_{3 \mathrm{LSB}}{ }^{+}{ }_{\text {max }}$ from the level of the first swingby. The second swingby options with $C_{3 \mathrm{LSB}}{ }^{+}{ }_{\max }>2 \mathrm{~km}^{2} / \mathrm{s}^{2}$ steadily occur after 70 days.

It has been concluded that $J$ increase at $\mathrm{S} 1$ and $\alpha$ approaching $80^{\circ}$ at $\mathrm{S} 2$ contribute to increasing $C_{3 \mathrm{LSB}}{ }^{+}$max. The $\mathrm{S} 1$ and $\mathrm{S} 2$ states are plotted on the $\alpha-J$ plane in Fig. 14 . It can be seen that there are some $\mathrm{S} 2^{-}$states with $\alpha$ near $80^{\circ}$, but rare states on the upper side where $J$ and $\varepsilon$ are high. Because high-

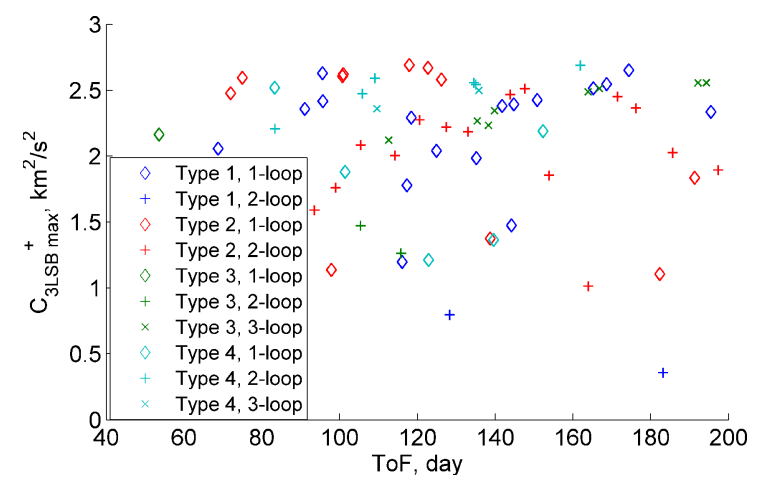

Fig. 13. $C_{3 \mathrm{LSB}}{ }^{+}{ }_{\text {max }}$ of the second swingby vs ToF.

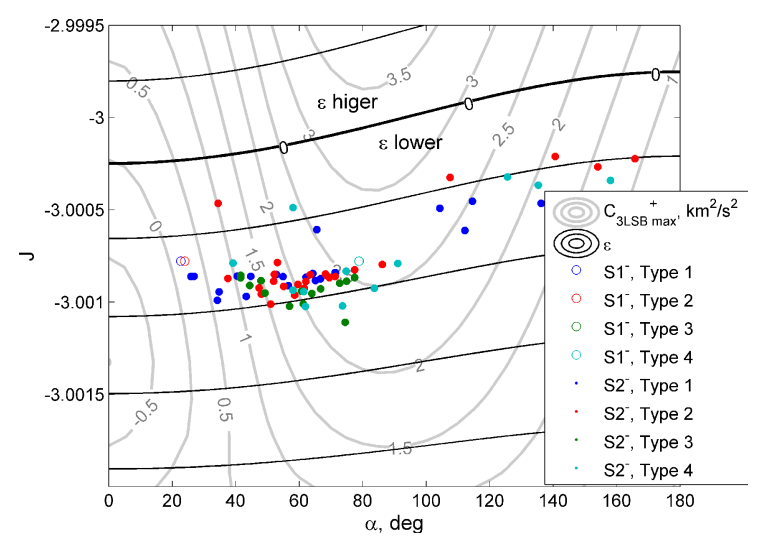

Fig. 14. $C_{3 \mathrm{LSB}}{ }^{+}{ }_{\text {max }}, \varepsilon, \mathrm{S} 1^{-}$and corresponding $\mathrm{S} 2^{-}$states on the $\alpha-J$ plane. (The $\mathrm{S}^{-}$of Type 3 and Type 4 overlap.)

$\varepsilon$ orbits have long periods, within a finite $T o F$, the chance of re-encounter is relatively rare. The upper limit of $3.3 \mathrm{~km}^{2} / \mathrm{s}^{2}$ for double swingbys is given at $\varepsilon=0$ without specifying the condition of $\mathrm{S} 1$ (i.e., lunar phase, $v_{\infty}$ and $\varphi^{-}$). A small distribution of $\mathrm{S} 1$ conditions can lead to transfers with $\varepsilon \approx 0$ within 200 days. Since there are only four specific S1 conditions using manifold transfer, the practical maximum $\left(2.7 \mathrm{~km}^{2} / \mathrm{s}^{2}\right)$ is shown not close to the theoretical limit. On the other hand, McElrath et al. ${ }^{14)}$ have computed double lunar swingbys for various $\mathrm{S} 1$ conditions within seven months $\left(\approx 200\right.$ days) and shown that the maximum $C_{3 \mathrm{LSB}}$ is $3.25 \mathrm{~km}^{2} / \mathrm{s}^{2}$. In an experiment of extending $T o F$, the results of which are not presented in this paper, an S2 solution with $C_{3 \mathrm{LSB}}{ }^{+}{ }_{\text {max }}$ of $3.23 \mathrm{~km}^{2} / \mathrm{s}^{2}$ can be found for the $\mathrm{S} 1$ of Type 2 in around 300 days, which is close to the limit of $3.3 \mathrm{~km}^{2} / \mathrm{s}^{2}$ 

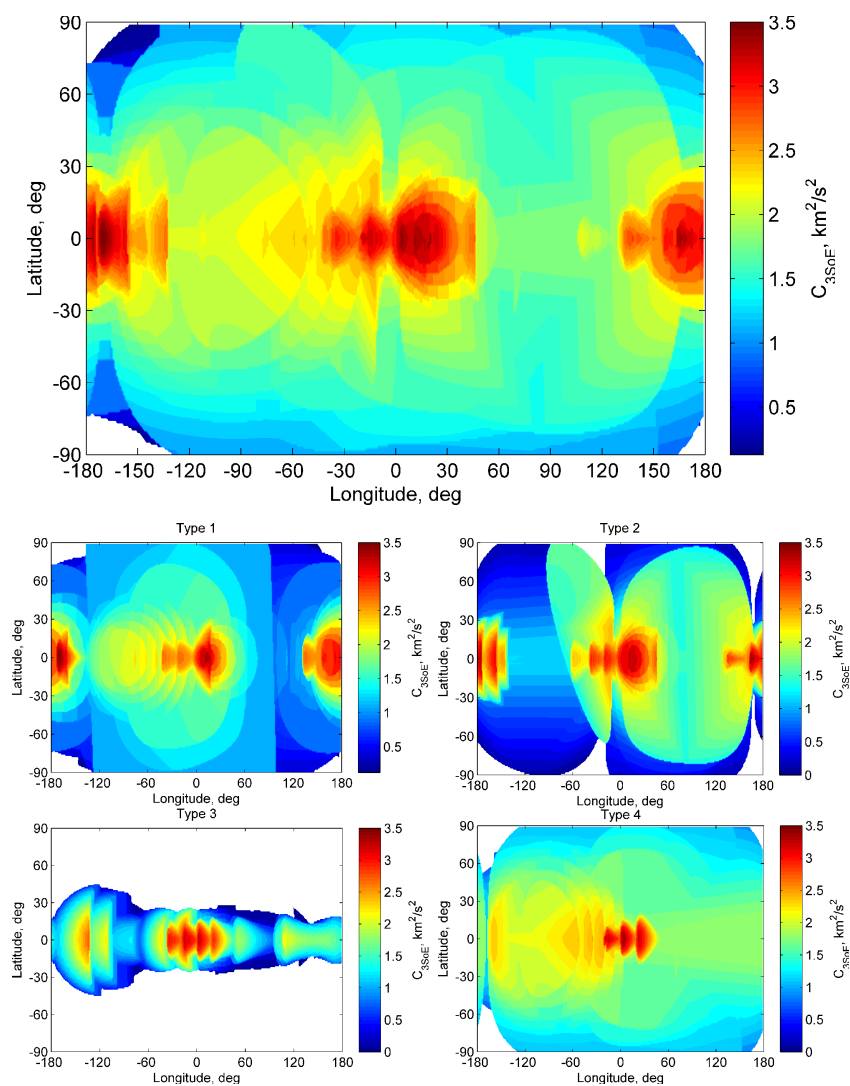

Fig. 15. The maximum $C_{3 \mathrm{SoE}}$ and the escape direction for the second swingbys of all types (uppermost) and for each type (lower).

(see Fig. 9 for the value of 2 nd swingby for Type 2 at $\left.A_{z}=4 \times 10^{5} \mathrm{~km}\right)$. In another experiment with $A_{z}=1 \times$ $10^{5} \mathrm{~km}$, an S2 solution with $C_{3 \mathrm{LSB}}{ }^{+}{ }_{\max }$ of $2.6 \mathrm{~km}^{2} / \mathrm{s}^{2}$ can be found for the $\mathrm{S} 1$ of Type 1 in 70 days, which is consistent with the theoretical limit (see Fig. 9). This is because the $C_{3 \mathrm{LSB}}{ }^{+}{ }_{\text {max }}$ level of $2.6 \mathrm{~km}^{2} / \mathrm{s}^{2}$ can be accomplished by transfers with moderate energy and flight time, which is likely to occur for any condition of S1.

In addition, it is interesting to compare the upper limits of $C_{3 \mathrm{LSB}}{ }^{+}{ }_{\text {max }}$ obtained from the swingby-Jacobi graph with the results in literature. A summary of the comparison is:

i) For short swingbys with unperturbed transfers, the upper limit of the $2.3 \mathrm{~km}^{2} / \mathrm{s}^{2}$ for $C_{3 \mathrm{LSB}}{ }^{+}{ }_{\text {max }}$ is consistent with the maximum $C_{3 \mathrm{LSB}}$ presented in Landau et al. ${ }^{8}$ and McElrath et al., ${ }^{14)}$ which compute various short double-lunar swingbys.

ii) For double swingbys with perturbed transfers, it has been mentioned that, within 200 days, the upper limit of $3.3 \mathrm{~km}^{2} / \mathrm{s}^{2}$ for $C_{3 \mathrm{LSB}}{ }^{+}{ }_{\text {max }}$ is consistent with McElrath et al. ${ }^{14)}$

iii) For multiple swingbys with perturbed transfers, within 200 days, the upper limit of $5 \mathrm{~km}^{2} / \mathrm{s}^{2}$ for $C_{3 \mathrm{LSB}}{ }^{+}$max is also consistent with McElrath et al., ${ }^{14)}$ which shows that the maximum $C_{3 \mathrm{LSB}}$ achieved by various triple swingby is $4.7 \mathrm{~km}^{2} / \mathrm{s}^{2}$.

With many S2 solutions derived from the S1 conditions, the escape directions should be further broadened. Again, the post-swingby states are propagated to the SoE. The escape directions and the maximum $C_{3 \mathrm{SoE}}$ in these directions are printed in Fig. 15. A $C_{3 \mathrm{SoE}}$ greater than $2 \mathrm{~km}^{2} / \mathrm{s}^{2}$ is generally available at all longitudes. In an SEP mission, the SEP can be used to steer the spacecraft back to Earth for gravity assist and meanwhile change the $v_{\infty}$ with respect to the Earth for an Earth gravity assist. The technique is called as the Electric Propulsion Delta-V Earth Gravity Assist (EDVEGA). ${ }^{16)}$ Landau et al. ${ }^{8)}$ has shown that an escape $C_{3}$ of $2 \mathrm{~km}^{2} / \mathrm{s}^{2}$ can be well used and greatly increased by applying the EDVEGA with a modern SEP, enabling missions to targets as far as Jupiter. Therefore, the $C_{3}$ achieved by the concerned escape strategy would be significant in establishing a deep-space mission.

\section{Conclusions}

Motivated by the increasing interest in halo orbit missions, the Earth escape strategy of utilizing the unstable manifold and lunar swingbys is proposed and analyzed. First, the four types of manifold-guided lunar encounters are obtained. Two types of them can lead to effective lunar gravity assists and a maximum $C_{3 \mathrm{LSB}}{ }^{+}$of $2.6 \mathrm{~km}^{2} / \mathrm{s}^{2}$ with respect to the Earth.

As the $v_{\infty}$ of the encounters of Type 1 and Type 2 are not high enough for the lunar swingbys to effectively increase $C_{3 \mathrm{LSB}}$, subsequent lunar swingbys utilizing solar tidal force to change the $v_{\infty}$, and thus the maximum achievable $C_{3 \mathrm{LSB}}{ }^{+}$, are discussed. The swingby-Jacobi graph is presented in this paper. Without numerically solving various Moon-to-Moon transfers, it reveals that the theoretical upper limits of the $C_{3 \mathrm{LSB}}$ achieved by short swingbys without Sun-perturbed transfers, double and a larger number of swingbys are $2.3 \mathrm{~km}^{2} / \mathrm{s}^{2}, 3.3 \mathrm{~km}^{2} / \mathrm{s}^{2}$ and $5 \mathrm{~km}^{2} / \mathrm{s}^{2}$, respectively. Within a $T o F=200$ days, the numerically solved second swingbys for the four types of manifold-guided encounter can achieve a maximum $C_{3 \mathrm{LSB}}$ of $2.7 \mathrm{~km}^{2} / \mathrm{s}^{2}$ for $A_{z}=4 \times 10^{5} \mathrm{~km}$. In particular, for Type 1 and Type 2, allowing another 75-95 days to perform a second swingby, the $C_{3 \mathrm{LSB}}$ can be increased to the practical maximum. Therefore, for the $\mathrm{S} 1$ with low $v_{\infty}, C_{3 \mathrm{LSB}}$ can be efficiently increased by applying a second lunar swingby.

The lunar swingby option may require a longer flight time to achieve Earth escape than the direct manifold escape. However, the swingby option that lead to considerable $C_{3}$ largely broadens the choices for a direct visit to heliocentric bodies, as well as enhances the EDVEGA technique, to which attention should be paid when extended missions for halo orbits are considered.

This study assumes the halo orbit mission has been prephased for a lunar encounter. However, for general extended missions, the halo orbit mission should not be pre-phased for a future destination. Because the use of unstable manifolds along with lunar gravity assists is shown advantageous, follow-up work will investigate the phasing cost.

\section{Acknowledgments}

This work was carried out when the first author was staying at ISAS/JAXA as a joint research fellow. The first author appreciates the resources provided by JAXA, especially the helpful advice from Dr. Stefano Campagnola and Dr. Takanao Saiki. The first author also acknowledges the support of her current workplace, Technology 
and Engineering Center for Space Utilization, Chinese Academy of Sciences, for finalizing this paper.

\section{References}

1) Villac, B. F. and Scheeres, D. J.: Escaping Trajectories in the Hill Three-Body Problem and Applications, J. Guid. Control Dynam., 26 (2003), pp. 224-232.

2) Nakamiya, M., Yamakawa, H., Scheeres, D. J., and Yoshikawa, M.: Interplanetary Transfers between Halo Orbits: Connectivity between Escape and Capture Trajectories, J. Guid. Control Dynam., 33 (2010), pp. 803-813.

3) Mingotti, G., Topputo, F., and Bernelli-Zazzera, F.: Earth-Mars Transfers with Ballistic Escape and Low-Thrust Capture, Celest. Mech. Dynam. Astron., 110 (2011), pp. 169-188.

4) Farquhar, R.: The Flight of ISEE-3/ICE: Origins, Mission History, and a Legacy, J. Astronaut. Sci., 49 (2001), pp. 23-73.

5) Kawaguchi, J., Yamakawa, H., Uesugi, T., and Matsuo, H.: On Making Use of Lunar and Solar Gravity Assists in LUNAR-A, PLANET-B Missions, Acta Astronaut., 35 (1995), pp. 633-642.

6) Campagnola, S., Jehn, R., and Corral, C.: Design of Lunar Gravity Assist for the BepiColombo Mission to Mercury, Adv. Astronaut. Sci., 119 (2004), pp. 427-442.

7) Guzman, J. J., Dunham, D. W., Sharer, P. J., Hunt, J. W., Ray, C. J., Shapiro, H. S., Ossing, D. A., and Eichstedt, J. E.: STEREO Mission Design Implementation, 20th International Symposium on Space Flight Dynamics, Annapolis, Maryland, USA, 2007.

8) Landau, D., McElrath, T. P., Grebow, D., and Strange, N. J.: Efficient Lunar Gravity Assists for Solar Electric Propulsion Missions, Adv. Astronaut. Sci., 143 (2012), pp. 917-934.

9) Uesugi, K.: Space Odyssey of an Angel: Summary of the HITEN's Three Years Mission, Adv. Astronaut. Sci., 84 (1993), pp. 607-621.

10) Folta, D. C., Woodard, M., Howell, K., Patterson, C., and Schlei, W.: Applications of Multi-body Dynamical Environments: The ARTEMIS Transfer Trajectory Design, Acta Astronaut., 73 (2012), pp. 237-249.

11) Kawakatsu, Y.: Destiny Mission Overview: A Small Satellite Mission for Deep Space Exploration Technology Demonstration, Adv. Astronaut. Sci., 146 (2012), pp. 727-739.

12) Howell, K. C.: Three-Dimensional, Periodic, "Halo" Orbits, Celest. Mech., 32 (1984), pp. 53-71.

13) Koon, W. S., Lo, M. W., Marsden, J. E., and Ross, S. D.: Dynamical Systems, the Three-Body Problem and Space Mission Design, Marsden Books (Online book, http://www.cds.caltech.edu/ marsden/ volume/missiondesign/KoLoMaRo_DMissionBook_2011-04-25.pdf) 2011, pp. 174-175.

14) McElrath, T. P., Lantoine, G., Landau, D., Grebow, D., Strange, N., Wilson, R., and Sims, J.: Using Gravity Assists in the Earth-Moon System as a Gateway to the Solar System, Global Space Exploration Conference, Washington DC, USA, 2012.

15) Lantoine, G. and McElrath, T. P.: Families of Solar-Perturbed Moonto-Moon Transfers, AAS/AIAA Space Flight Mechanics Meeting, Santa Fe, USA, 2014.

16) Kawaguchi, J.: Solar Electric Propulsion Leverage: Electric DeltaVEGA (EDVEGA) Scheme and Its Application, AAS/AIAA Space Flight Mechanics Meeting, 2001.

17) Szebehely, V.: Theory of Orbits: The Restricted Problem of Three Bodies, Academic Press, New York, San Francisco, London, 1967, pp. 134-139.

\section{Appendix}

The Circular Restricted Three-Body Problem (CR3BP) assumes two primary bodies moving in a circular orbit about their barycenter. The mass of the third body is negligible

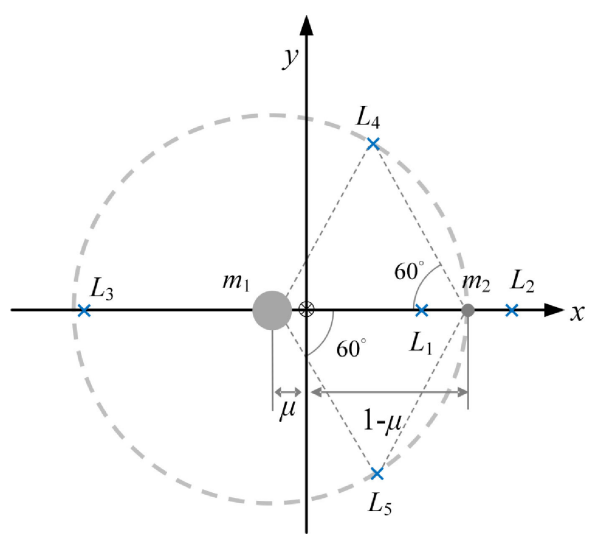

Fig. A.1. Circular restricted three-body system and Lagrangian points.

compared to the masses of the two primaries. The two primaries can be the Sun and Earth, the Earth and Moon, etc. The rotating coordinate system with the barycenter at the origin and the primaries fixed on the $x$-axis is chosen to describe the motion of the third body. For convenience, the angular velocity of the rotating frame, total mass and distance between the two primaries are normalized to $1 . \mu$ is the ratio of the mass of the secondary primary body $m_{2}$ to the mass of the first primary body $m_{1}$. The $m_{1}$ and $m_{2}$ become $1-\mu$ and $\mu$ in the normalized model. Then, the coordinates of the first and secondary bodies are $[-\mu, 0,0]$ and $[1-\mu, 0,0]$, respectively, as shown in Fig. A.1. The equations of motion of the third body are:

$$
\begin{gathered}
\ddot{x}-2 \dot{y}=\partial U / \partial x \\
\ddot{y}+2 \dot{x}=\partial U / \partial y \\
\ddot{z}=\partial U / \partial z
\end{gathered}
$$

where the pseudo-gravitational potential $U$ in the system is

$$
U=\left(x^{2}+y^{2}\right) / 2+(1-\mu) / r_{1}+\mu / r_{2}
$$

where

$r_{1}=\sqrt{(x+\mu)^{2}+y^{2}+z^{2}}, \quad r_{2}=\sqrt{(x-1+\mu)^{2}+y^{2}+z^{2}}$

The system has an integral of motion,

$$
J=2 U-\left(\dot{x}^{2}+\dot{y}^{2}+\dot{z}^{2}\right)
$$

which is constant and is called the Jacobi integral.

\section{Equilibrium points}

Szebehely ${ }^{17)}$ has given the details of resolving the five equilibrium points from the equations of motion. They are also referred to as Lagrangian points labeled as $L_{1}, \cdots, L_{5}$. The three collinear points, $L_{1}, L_{2}$ and $L_{3}$, are unstable. The geometries of the five equilibrium points are depicted in Fig. A.1. 